\title{
Radiation-damage-assisted ferroelectric domain structuring in magnesium-doped lithium niobate
}

\author{
L. Jentjens • K. Peithmann • K. Maier • H. Steigerwald • \\ T. Jungk
}

Received: 19 December 2008 / Revised version: 17 March 2009 / Published online: 3 April 2009

(C) The Author(s) 2009. This article is published with open access at Springerlink.com

\begin{abstract}
Irradiation of 5\% magnesium-doped lithium niobate crystals $\left(\mathrm{LiNbO}_{3}: \mathrm{Mg}\right)$ with high-energy, low-mass ${ }^{3} \mathrm{He}$ ions, which are transmitted through the crystal, changes the domain reversal properties of the material. This enables easier domain engineering compared to non-irradiated material and assists the formation of small-sized periodically poled domains in $\mathrm{LiNbO}_{3}: \mathrm{Mg}$. Periodic domain structures exhibiting a width of $\approx 520 \mathrm{~nm}$ are obtained in radiation-damaged sections of the crystals. The ferroelectric poling behavior between irradiated and non-treated material is compared.
\end{abstract}

PACS $78.20 \cdot 42.65$

\section{Introduction}

Ferroelectric lithium niobate crystals $\left(\mathrm{LiNbO}_{3}\right)$ are of tremendous interest for applications in optics: the material is commonly used for integrated-optical devices such as waveguides [1], lasers [2], modulators [3], and couplers [4]. Special attention has been given to periodically poled lithium niobate crystals (PPLN) which exhibit domains with a periodically inverted polar axis: the periodic structure enables very efficient frequency doubling via quasi-phase-matching [5]. Using standard, congruently melting $\mathrm{LiNbO}_{3}$ crystals, however, has a crucial disadvantage: incident light can change the refractive index locally,

L. Jentjens $\cdot$ K. Peithmann $(\bowtie) \cdot$ K. Maier

Helmholtz-Institut für Strahlen- und Kernphysik, Universität

Bonn, Nussallee 14-16, 53115 Bonn, Germany

e-mail: peithmann@physik.uni-bonn.de

H. Steigerwald · T. Jungk

Physikalisches Institut, Universität Bonn, Wegelerstr. 8,

53115 Bonn, Germany yielding a distortion of the light beam profile, an effect known as the photorefractive effect or optical damage [6]. Doping $\mathrm{LiNbO}_{3}$ with magnesium $\left(\mathrm{LiNbO}_{3}: \mathrm{Mg}\right)$ at doping levels of $5 \mathrm{~mol} \%$ in congruently melting material suppresses the photorefractive effect very efficiently [7]. However, it is difficult to structure the ferroelectric domains of crystals containing such a high percentage of $\mathrm{Mg}$ with standard methods used for undoped material. Hence, methods improving the poling feasibility of $\mathrm{LiNbO}_{3}: \mathrm{Mg}$ are highly desired. For this purpose, ultraviolet light, helping small-sized domains to grow, has been utilized [8, 9]. In this paper we present a different approach: the crystals are treated with low-mass, high-energy ions where the ion energy is large enough that they are transmitted through the full material thickness. On their path through the crystal, radiation damage takes place influencing domain reversal parameters such as the coercive field $E_{\mathrm{C}}$ [10]. However, it is questionable whether this change of $E_{\mathrm{C}}$ supports the formation of smallsized, periodically poled ferroelectric domains directly: it is rather expected that small-sized domains become possible by defects stabilizing the domain walls. Because irradiation with ions induces additional defects in the crystals, we have investigated the impact of radiation damage on the formation of small-sized ferroelectric domain structures, comparing irradiated with non-treated crystals.

\section{Experimental methods}

Magnesium-doped lithium niobate crystals supplied by Yamaju Ceramics, Japan, doped with $5 \mathrm{~mol} \% \mathrm{Mg}$, were prepared in $z$-cut geometry with a size of $(x \times y \times z)=16 \times$ $15 \times 0.5 \mathrm{~mm}^{3}$ by cutting them from a single wafer, and the large $z$-surfaces were polished to optical quality. Irradiation treatments of the crystals were carried out at the cyclotron of 
the Helmholtz-Institute for Radiation and Nuclear Physics at the University of Bonn. Here doubly-ionized ${ }^{3} \mathrm{He}$ ions with an energy of $41 \mathrm{MeV}$ were provided which were incident on the crystal's $z$-surface. The range of the ions, i.e. the propagation length of the ions through $\mathrm{LiNbO}_{3}: \mathrm{Mg}$ at that energy, was $530 \mu \mathrm{m}$ as calculated by SRIM simulations [11]; hence the ions can be considered to be transmitted through the entire crystal thickness $(d=500 \mu \mathrm{m})$. The samples were mounted onto an aluminum heat sink using heat conductive paste. The ion beam was shaped by a graphite slit with dimensions of $2 \times 8 \mathrm{~mm}^{2}$; a total ion current of $60 \mathrm{nA}$ yields an ion fluence per second of $1.7 \times 10^{16}$ ions $/ \mathrm{m}^{2} \mathrm{~s}$. Typical irradiation treatments lasted between 20 and $70 \mathrm{~min}$. The irradiated area was located in the center of the $z$-surface of the crystal.

In order to fabricate Mg-doped PPLN the crystals were coated after the irradiation process with a grating-like structured photoresist pattern (AZ1512) on the $+z$-surface. The resist had a thickness of $1.5 \mu \mathrm{m}$ and was structured with a period of either 10 or $18 \mu \mathrm{m}$, exhibiting a duty cycle of $1: 1.5$, or a period of $5.3 \mu \mathrm{m}$ with a duty cycle of $1: 4.3$. For domain reversal, the crystal was clamped between two fused silica glass plates with silicone o-rings. The space within the o-rings was filled with liquid electrodes $[8,12]$. Subsequently, high voltage was applied to the $z$-surfaces. We used high voltage pulses (up to $5 \mathrm{kV}$ ), generated by a high voltage amplifier (TREK 20C) which was controlled by a function generator; the pulse duration was typically $600 \mathrm{~ms}$. During the poling experiments, the redistribution of compensation charges on the crystal surface was monitored. The application of multiple voltage pulses yielded structured domain inversion.

Spatially-resolved domain reversal can also be achieved using a scanning force microscope: applying a voltage to the tip and thus generating an electric field high enough to exceed $E_{\mathrm{C}}$ of the material locally reverses the spontaneous polarization. Even if the field at the apex of the tip easily exceeds $E_{\mathrm{C}}$, stable domains can be written only if the applied voltage is above a threshold value yielding fields at the apex much higher than $E_{\mathrm{C}}$. To obtain sufficient electric fields throughout the whole crystal thickness, thin crystals of a few tens of micrometers are necessary. Thus, we prepared thin slabs out of our irradiated samples by grinding and polishing the back surface, removing $\approx 80 \mu \mathrm{m}$ of the material, and subsequent bonding the surface to indium tin oxide coated glass substrates. Next the front surface was ground and polished until a thin layer of $\approx 50 \mu \mathrm{m}$ remained. This procedure ensured that ion-irradiated material was obtained from the inner regions of the crystal in order to have a good compromise between sufficient radiation damage without being too close to the Bragg peak, which yields too strong low-energy ion-damage processes.

Ferroelectric domains in $\mathrm{LiNbO}_{3}$ crystals can be visualized by several methods [13]: here the crystals were etched in hydrofluoric acid (HF) which converted a domain pattern into a corresponding topographical profile that could be observed by an optical microscope. Alternatively, we used piezoresponse force microscopy (PFM) to visualize the domain structures without etching [14]. For PFM a scanning force microscope is operated in contact mode with an additional alternating voltage applied to the tip. In piezoelectric samples this voltage causes thickness changes and therefore vibrations of the surface, which lead to oscillations of the cantilever that can be read out with a lock-in amplifier.

\section{Results}

For the experiments, we have prepared three different samples. Sample A has been irradiated with a total ion fluence of $2 \times 10^{19}$ ions $/ \mathrm{m}^{2}$ (irradiation time $\approx 20 \mathrm{~min}$ ) and has been coated with a mask having a period of $18 \mu \mathrm{m}$. An optical microscope image of the HF-etched sample is shown in Fig. 1 . The previously ion-irradiated region exhibited periodically poled ferroelectric domains with a period of $18 \mu \mathrm{m}$ whereas in the non-irradiated part of the sample distinctly fewer domains were observed. The inset of Fig. 1 displays a part of the crystal right at the edge between irradiated and non-irradiated regions, where the differences in the domain formation process can be seen.

Figure 2(a) shows an image of sample B which has been treated with a total ion fluence of $7 \times 10^{19}$ ions $/ \mathrm{m}^{2}$ (irradiation time $\approx 70 \mathrm{~min}$ ). In the subsequent poling experiment a mask with a period of $5.3 \mu \mathrm{m}$ was used. The mask covered the entire crystal area displayed in Fig. 2(a), consisting of an

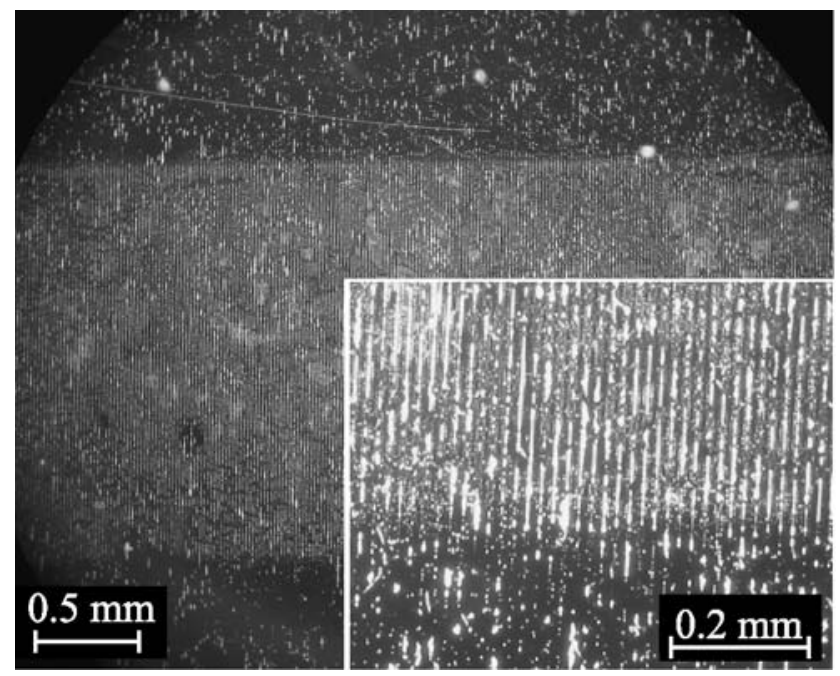

Fig. 1 Optical microscope image of the $+z$-surface of HF-etched sample A after a domain reversal experiment using an $18 \mu \mathrm{m}$ period mask. In the previously ion-irradiated regions a much stronger domain reversal process was observed compared to the non-treated sections of the crystal. The inset shows a part of the crystal under larger magnification, where the periodicity of the mask has been faithfully reproduced 
Fig. 2 Optical microscope image (a) of the HF-etched sample B after domain reversal experiment using a $5.3 \mu \mathrm{m}$ mask; Fig. 2(b) shows a part of the crystal under larger magnification. In the irradiated mask periodicity of $5.3 \mu \mathrm{m}$ were present (upper part of (b)), whereas in the non-treated regions larger, non-periodic structured domains were observed (lower part of (b)) sections domains exhibiting the
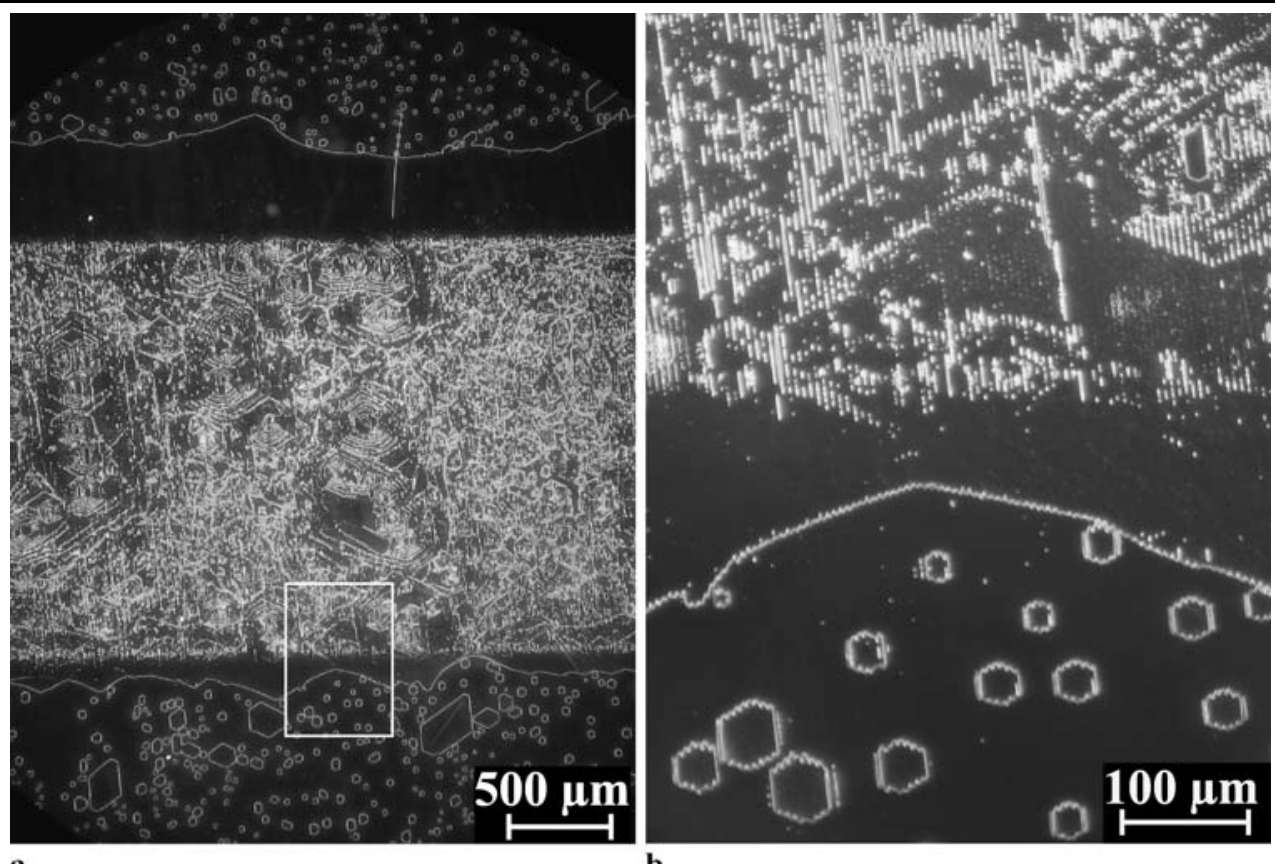

b

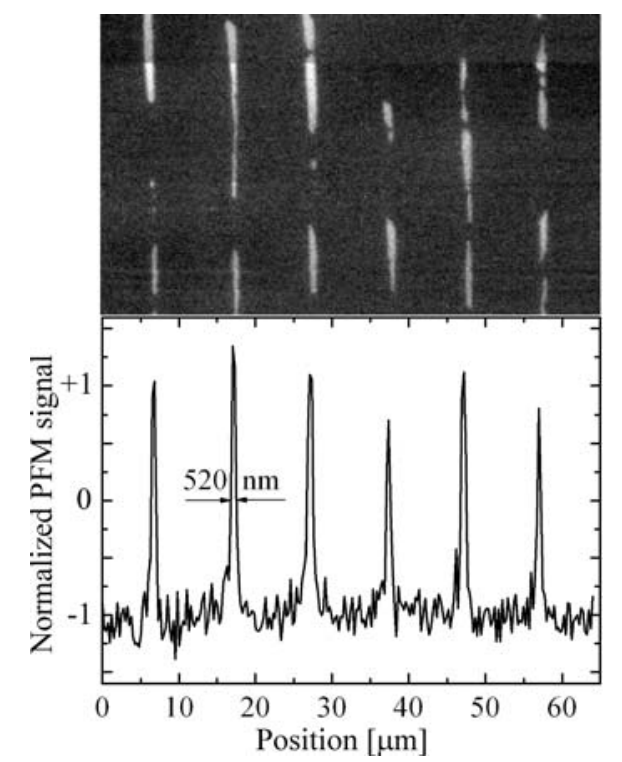

irradiated region (middle stripe in Fig. 2(a)) and non-treated parts (upper and lower parts in Fig. 2(a)). Figure 2(b) shows a magnification of the indicated area of Fig. 2(a). Two results have to be emphasized: in the irradiated region, small-sized ferroelectric domains appeared clearly reflecting the mask periodicity. In the non-irradiated part of the crystal, shown in the lower part of Fig. 2(b), we found that domains, when present, exhibited the typical underlying $3 \mathrm{~m}$ crystal symmetry of $\mathrm{LiNbO}_{3}[15]$ but they did hardly reflect the periodicity of the mask. In particular, most of the domains in the nontreated region were much larger than $5 \mu \mathrm{m}$, and only at their edges could the periodicity of the mask be seen.

In Fig. 3 we show a typical PFM image of the $+z$-surface of sample C (fluence $6 \times 10^{19} \mathrm{ions} / \mathrm{m}^{2}$, irradiation time $\approx 60 \mathrm{~min}$, mask period $10 \mu \mathrm{m}$ ). Small domain stripes with a width of $\approx 520 \mathrm{~nm}$ were observed. This corresponds to a domain duty cycle of $1: 19$, compared to the photomask duty cycle of $1: 1.5$. Because PFM can distinguish the direction of the $z$-axis with respect to the surface, we can identify that dark regions in Fig. 3 are reversed domains, whereas the bright stripes belong to the non-reversed domains. Hence, in crystal $\mathrm{C}$ almost complete domain reversal has taken place.

We also performed local poling experiments using diamond coated tips of $60 \mathrm{~nm}$ nominal radius of a scanning force microscope (model SMENA from NT-MDT Co., Russia). First, the minimum voltage (exposure time $5 \mathrm{~min}$ ) to write domains stable for at least 14 hours was identified in both the irradiated and the non-irradiated parts of the crystal. As the electric field was inhomogeneous and strongly dependent on the exact shape of the tip we only give the poling voltages (see Table 1). The errors are given by the
Fig. 3 Upper part: Typical PFM image of the surface of sample C. Lower part: A line scan is shown, together with an indication of the FWHM of the domains

standard deviation calculated from the poling voltages at 3-5 different positions.

Furthermore, the domain growth characteristics were analyzed by poling with a fixed voltage of $\pm 100 \mathrm{~V}$ for $10 \mathrm{~min}$ and subsequently imaging the written domains via PFM. The results are shown in Fig. 4: most prominent is the difference between the poling results in the virgin part of the crystal (Fig. 4(a)) and the reversed or irradiated region respectively (Fig. 4(b-d)). Moreover, in the ${ }^{3} \mathrm{He}$-irradiated re- 
Table 1 Minimum poling voltages to write stable domains and domain sizes poled with the PFM tip in the different parts of the $\mathrm{LiNbO}_{3}: \mathrm{Mg}$ crystal
Crystal section

Minimum poling voltage

Domain size

(5 min exposure time)

$( \pm 100 \mathrm{~V}, 10 \mathrm{~min})$

Non-irradiated, non domain-reversed

Non-irradiated, domain-reversed

Irradiated, non domain-reversed

Irradiated, domain-reversed

$-(100 \pm 4) \mathrm{V}$

$0.5 \mu \mathrm{m}^{2}$

$+(55 \pm 2) \mathrm{V}$

$1.8 \mu \mathrm{m}^{2}$

$-(40 \pm 3) \mathrm{V}$

$2.6 \mu \mathrm{m}^{2}$

$+(45 \pm 2) \mathrm{V}$

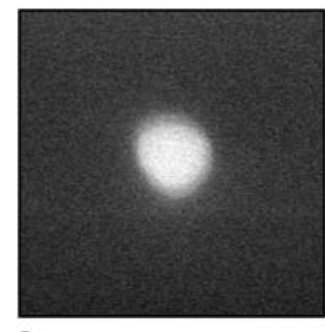

a

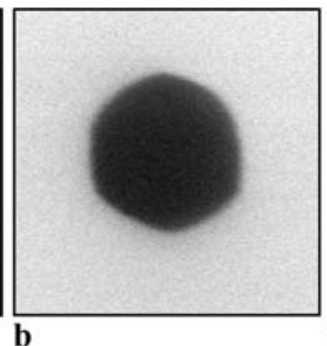



c

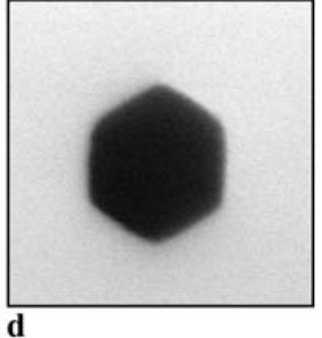

Fig. 4 PFM images $\left(3 \times 3 \mu \mathrm{m}^{2}\right.$ size $)$ of domains poled by applying voltages $( \pm 100 \mathrm{~V})$ with the help of the PFM tip (10 min exposure time) in a $50 \mu \mathrm{m}$ thickness $\mathrm{LiNbO}_{3}: \mathrm{Mg}$ layer: the domains poled in the non-irradiated part $(\mathbf{a}, \mathbf{b})$ were less hexagonal as compared to domains poled in ${ }^{3} \mathrm{He}$-irradiated region (c, d). The bright (dark) area corresponds to the $+z-(-z)$-surface of the crystal

The domain growth characteristics in the different regions of the crystal vary significantly as can be deduced from the PFM measurements. In the regions with higher defect concentrations, i.e. in the domain-reversed or in the ${ }^{3} \mathrm{He}$-irradiated part, the poling starts at about half the voltage as compared to the virgin crystal. Comparing the minimum voltages to write stable domains in the previously poled region (Table 1) we find that the irradiated part can be poled with about $20 \%$ lower voltages than the non-irradiated part, hence, the coercive field is significantly lowered there. This is in good agreement with recently published results [10]. However, it is not possible to deduce the exact values of coercive fields as the detailed poling process via the inhomogeneous field of the tip is still under discussion [17-19]. Nonetheless, PFM imaging is not hampered by the inhomogeneous field [20], yielding distinct differences in the poling process: in the irradiated region of the crystal the domains always grow in regular shaped hexagons whereas in the non-irradiated part the domain structures are distinctly less hexagonally shaped and rather circular. According to the defect dipole model for $\mathrm{LiNbO}_{3}$ [21] the domain wall motion and stabilization can be explained by pinning at defects. Thus, the hexagons are very likely stabilized via defects introduced by the ${ }^{3} \mathrm{He}$ ion irradiation. To refine our findings a series of poling experiments with various $\mathrm{LiNbO}_{3}: \mathrm{Mg}$ crystals with, e.g., different thickness or different irradiation fluences, is desirable.

We consider our experiments as a proof-of-principle showing that ion irradiation has a significant, positive impact on the poling properties of $\mathrm{LiNbO}_{3}: \mathrm{Mg}$; however, more effort has to be taken to improve the quality of the peri- 
odically structured crystals. The ion fluences used in this work were initially chosen values, according to established ion fluences used for preparation of refractive index modulations or changes in the coercive field [10], which are not optimized. It will be necessary to investigate the dependence of the effects on the ion fluence. In particular, it will be interesting if lower ion fluences can still yield reasonable results and if there exists a saturation behavior for very strong ion exposure.

Please note that quite similar results can be achieved using UV light illuminating the crystals during the domain reversal process $[9,12,22,23]$. This method also yields surface nano-structured domains in $\mathrm{LiNbO}_{3}: \mathrm{Mg}$. The advantages of ion-treated material can be summarized as follows: the process of irradiation can be separated from the domain engineering, which is different from the UV light-assisted poling, where simultaneous illumination during the application of high voltage is required in the case of $\mathrm{LiNbO}_{3}: \mathrm{Mg}$ [24]. Furthermore, we expect that radiation-damaged material can be treated using the well established poling process steps with optimized electrode design, different to the UV process requiring special UV suitable masks with a minimum thickness limiting the resolution achievable. Standard masks can be made thinner yielding smaller structures. The similarity between UV illumination and ion exposure has been discovered before: both treatments yield a significant reduction of the coercive field $E_{\mathrm{C}}[8,10,25]$, which implies that changes in the electronic structure of the material are crucial for the poling dynamics.

\section{Conclusion}

Irradiation of magnesium-doped, optical damage resistant lithium niobate crystals with high-energy, low-mass ${ }^{3} \mathrm{He}$ ions assists the formation of small-sized ferroelectric domains, significantly supporting the fabrication of periodically poled crystals. This is an interesting alternative to UVassisted domain engineering, as it yields improved raw material for the periodic poling process of $\mathrm{LiNbO}_{3}$ either using standard poling techniques or direct writing concepts utilizing piezoelectric force microscopy, yielding components which are highly desired for applications in modern optics.

Acknowledgements Technical support of the cyclotron team of the Helmholtz-Institut, Universität Bonn, is highly appreciated. Financial support of the DFG (Grant FOR 557-R2) and the Deutsche Telekom $\mathrm{AG}$ is gratefully acknowledged.
Open Access This article is distributed under the terms of the Creative Commons Attribution Noncommercial License which permits any noncommercial use, distribution, and reproduction in any medium, provided the original author(s) and source are credited.

\section{References}

1. D. Kip, Appl. Phys. B 67, 131-150 (1998)

2. C. Becker, A. Greiner, T. Oesselke, A. Pape, W. Sohler, H. Suche, Opt. Lett. 23, 1194-1196 (1998)

3. V. Ramaswamy, M.D. Divino, R.D. Standley, Appl. Phys. Lett. 32, 644-646 (1978)

4. R.C. Alferness, R.V. Schmidt, E.H. Turner, Appl. Opt. 18, 4012 4016 (1979)

5. G.A. Magel, M.M. Fejer, R.L. Byer, Appl. Phys. Lett. 56, 234236 (1989)

6. A. Ashkin, G.D. Boyd, J.M. Dziedzic, R.G. Smith, A.A. Ballman, J.J. Levinstein, K. Nassau, Appl. Phys. Lett. 9, 72-74 (1966)

7. D.A. Bryan, R. Gerson, H.E. Tomaschke, Appl. Phys. Lett. 44, 847-849 (1984)

8. M.C. Wengler, B. Fassbender, E. Soergel, K. Buse, J. Appl. Phys. 96, 2816-2820 (2004)

9. C.E. Valdivia, C.L. Sones, S. Mailis, J.D. Mills, R.W. Eason, Ferroelectrics 340, 75-82 (2006)

10. L. Jentjens, H. Hattermann, K. Peithmann, M. Haaks, K. Maier, M. Kösters, J. Appl. Phys. 103, 034104 (2008)

11. J.F. Ziegler, J.P. Biersack, U. Littmark, The Stopping and Range of Ions in Solids (Pergamon, New York, 1985)

12. H. Steigerwald, F. Luedke, K. Buse, Appl. Phys. Lett. 94, 032906 (2009)

13. E. Soergel, Appl. Phys. B 81, 729-751 (2005)

14. T. Jungk, Á. Hoffmann, E. Soergel, Appl. Phys. Lett. 89, 163507 (2006)

15. R.S. Weis, T.K. Gaylord, Appl. Phys. A 37, 191-203 (1985)

16. T. Jungk, Á. Hoffmann, E. Soergel, New J. Phys. 10, 013109 (2008)

17. S. Bühlmann, E. Colla, P. Muralt, Phys. Rev. B 73, 214120 (2005)

18. Y. Kan, X. Lu, X. Wu, J. Zhu, Appl. Phys. Lett. 89, 262907 (2006)

19. A.L. Kholkin, I.K. Bdikin, V.V. Shvartman, N.A. Pertsev, Nanotechnology 18, 095502 (2007)

20. T. Jungk, Á. Hoffmann, E. Soergel, Appl. Phys. A 86, 353-355 (2007)

21. S. Kim, V. Gopalan, K. Kitamura, Y. Furukawa, J. Appl. Phys. 90, $2949(2001)$

22. A.C. Muir, C.L. Sones, S. Mailis, R.W. Eason, T. Jungk, Á. Hoffmann, E. Soergel, Opt. Express 16, 2336-2350 (2008)

23. C.L. Sones, A.C. Muir, Y.J. Ying, S. Mailis, R.W. Eason, T. Jungk, Á. Hoffmann, E. Soergel, Appl. Phys. Lett. 92, 072905 (2008)

24. S. Mailis, C.E. Valdivia, C.L. Sones, A.C. Muir, R.W. Eason, in Proc. CLEO Europe, p. 380 (2007)

25. M.C. Wengler, U. Heinemeyer, E. Soergel, K. Buse, J. Appl. Phys. 98, 064104 (2005) 